\title{
Slow-Rate Utility-Based Resource Allocation in Wireless Networks
}

\author{
Peijuan Liu, Randall Berry, Michael L. Honig \\ ECE Department, Northwestern University \\ 2145 Sheridan Road, Evanston, IL 60208 USA \\ \{peijuan,rberry,mh\}@ece.nwu.edu
}

\author{
Scott Jordan \\ ECE Department, University of California, Irvine \\ 544D Engg. Tower, Irvine, CA 92697-2625 \\ sjordan@uci.edu
}

\begin{abstract}
We consider forward-link power allocation in a wireless network with stochastically varying data requests. We assume a user's service preferences are specified via a utility function that depends on the received data rate. The allocation of power across users is studied, where this allocation may depend on both a user's channel and utility. The objective is to maximize the time-averaged utility rate subject to a stochastic total power constraint at the transmitter. For a large, heavily loaded network, we introduce a Gaussian approximation for the total transmitted power, which is used to decompose the power constraint into three more tractable constraints. We present a solution to this problem that is a combination of admission control and pricing of power. The optimal trade-off between these approaches is characterized. Numerical examples are given to illustrate these ideas.
\end{abstract}

\section{INTRODUCTION}

The efficient allocation of radio resources, such as transmission power, is essential for supporting diverse applications over wireless networks. This paper investigates resource allocation for the forward link in a wireless network using a utility-based approach, where a user's service preference is specified by a single quality indicator or utility function. One advantage of such an approach is that different utility functions can be used to accommodate a wide range of traffic flows under a single framework. Also, utility functions can be used to capture many common definitions of "fairness" within a network [6].

Utility-based resource allocation has recently received attention both for wire-line [4], [3], [2] and wireless networks[1], [8], [5]. Related work addressing the forward link in a CDMA network can be found in [5], where the problem of maximizing aggregate utility subject to constraints on available transmission power and spreading codes was studied. The solution to this problem can be interpreted in a pricing framework, where prices per unit power and per code are announced, and users maximize their surplus (utility minus cost). The optimal allocation of resources can be found by choosing the correct resource prices. In [5] and much of the other prior work, the focus is on allocating resources for a static situation, where the number of users is fixed and each user will fully utilize whatever resources it is allocated. In this paper, we consider a situation where traffic is dynamically changing over the time period in which resources are allocated. In this case, random traffic variations must be taken into account when allocating resources.

We consider a model where the base station's transmission power is allocated among the users. Transmission requests randomly arrive at the base station; each request contains a fixed

This work was supported by the Motorola-Northwestern Center for Telecommunications, and by NSF under grant CCR-9903055. amount of data. The rate at which a request is transmitted, and hence the time to serve a request, depends only on the power allocation and the user's channel. The utility a user derives is based on how fast the request is served. We study the problem of allocating transmission power to maximize the time average utility rate, given a constraint on the total power transmitted by the base station. Since the traffic is randomly varying, the total power transmitted by the base-station is a random process. We consider a stochastic constraint on this process, which limits the total power to be less than a given value with high probability. We characterize the solution to this problem for a system with a large number of users. This solution can be viewed in a pricing framework as in [5]; however, there are several fundamental differences. First, in addition to pricing, explicit admission control is also needed. Second, the price that is used is not a fixed price for the constrained resource, the transmission power; the price instead depends on the product of the transmission power and energy, resulting in a non-linear price for the required power.

Our focus is on the situation where traffic variations occur on a much faster time-scale than that over which resource allocation is done. Specifically, we assume power is allocated based on the users' channel gain and utility, and this assignment is fixed over the time period of interest. In particular, the power allocation does not depend on the instantaneous system state (e.g., the number of active requests), but only on the long term statistics of the system. An alternate approach would be to take into account the current system state and reallocate resources at every arrival and departure. This type of approach has been studied in [2], via dynamic programming techniques. Clearly, allocating resources on a faster time-scale may improve the resulting utility rate. However, such an approach may not be feasible, due to various system constraints, and will require a more complicated allocation policy. Also, since the allocation considered here is not state dependent, a user receives a fixed utility upon admission. In contrast, with state dependent reallocations, the utility a user receives can vary depending on future events.

The rest of the paper is organized as follows. In Sect. II, we introduce a model for the forward link of a single cell. In Sect. III, we formulate a constrained optimization problem where the objective is to maximize the time-averaged utility rate subject to a stochastic constraint on the total power. In Sect. IV, a solution to this problem based on decomposing the power constraint into three more tractable constraints is presented. We then identify the system behavior under optimality. In Sect. V, we present numerical results illustrating these ideas. 


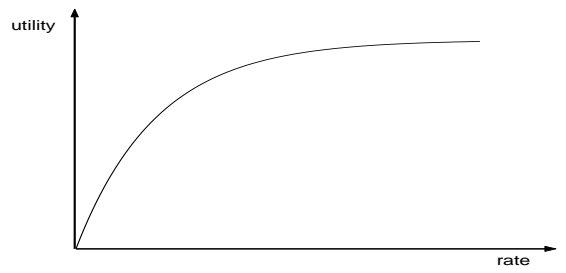

Fig. 1. Example utility function for data traffic.

\section{SySTEM MOdEL}

We describe a model for the forward link within a single cell, where the base station transmits simultaneously to all active users, and transmissions to different users are assumed to be orthogonal. For example, this models a CDMA system with orthogonal spreading codes. Suppose that a user is allocated transmission power $P$. The received Signal-to-Interference Plus Noise Ratio (SINR) for this user is given by $h P / \sigma^{2}$, where $h$ is the channel gain for that user and $\sigma^{2}$ is the total noise plus interference level. We assume that the received data rate for a user is a function of the received power, or equivalently received SINR; this relationship is given by $R(h)=\mathcal{C}(h P)$, where $\mathcal{C}(\cdot)$ is an increasing function.

User requests are modeled as arriving at the base station according to a Poisson process with overall rate $\lambda$. Each request consists of a unit of data with fixed length $L{ }^{*}$ Each data unit is referred to as a packet, however, this could also be a sequence of packets or a file depending on the situation. We consider a system with a large number of users, and assume each request corresponds to a new user. The channel gain of each user is assumed to be distributed on the interval $\mathcal{H}=\left(h_{\text {min }}, h_{\text {max }}\right)$, where $h_{\min } \geq 0$ and $h_{\max }<\infty$, with continuous density function, $f_{H}(h)$. This density can be used to model the users' geographic distribution within the cell, and also propagation effects such as random shadowing. The channel gain corresponding to each arrival is chosen independently according to this distribution and stays fixed during the entire transmission of the packet.

A utility function is associated with each request; this reflects a user's desired Quality of Service (QoS). We assume that utility depends only on the transmission rate $R$. Since each packet has a fixed length, this is equivalent to defining utility as a function of packet transmission time. In this paper, all users are assumed to have the same utility function, $U(R)$; however, this formulation can naturally be extended to cases with multiple utility classes. We assume that $U(0)=0$ and that $U(R)$ is increasing, concave and continuously differentiable with respect to $R$, for $R \geq 0$. These are common assumptions for so-called "elastic" traffic, which describes many data applications [7]. An example utility function is depicted in Fig. 1.

Since all users have the same utility function, the power allocated to a user depends only on the channel gain $h$. For each $h \in \mathcal{H}$, it will be useful to define the function $\tilde{U}(P(h))$, which relates the utility received by a user with channel gain $h$ to the transmitted power $P(h)$. This function is given by

$$
\tilde{U}(P(h))=U(\mathcal{C}(h P(h))) .
$$

\footnotetext{
${ }^{*}$ The following can be extended to the case where the length of each request is random, but we will not address this here.
}

Notice that $\tilde{U}(P(h))$ will be different for users with different channel gains even though these users have the same $U(R)$.

\section{Problem Formulation}

Our objective is to allocate transmission power to maximize the utility rate given a constraint on the total transmission power. A power allocation is specified by a function $P: \mathcal{H} \mapsto \mathbb{R}^{+}$that indicates the power used to transmit a packet to a user with channel gain $h \in \mathcal{H}$. If $P(h)=0$, the corresponding requests are considered blocked and not transmitted. If $P(h)>0$, the corresponding packets are transmitted with a transmission time given by

$$
T(h)=\frac{L}{\mathcal{C}(h P(h))} .
$$

For $i=1,2, \ldots$, let $H_{i}$ denote the channel gain of the $i$ th arrival, and let $N(t)$ denote the number of arrivals in the interval $[0, t)$. For a given power allocation, the time average utility rate is given by

$$
\lim _{T \rightarrow \infty} \frac{1}{T} \sum_{i=1}^{N(T)} \tilde{U}\left(P\left(H_{i}\right)\right)=\lambda \mathbb{E}_{H}\left\{\tilde{U}\left[P\left(H_{i}\right)\right]\right\},
$$

where the expectation is taken with respect to $f_{H}(h)$.

Let $\mathcal{A}(t)$ denote the set of active transmissions at time $t$. The total power transmitted at time $t$ can then be written as

$$
P_{\text {sum }}(t)=\sum_{i \in \mathcal{A}(t)} P\left(H_{i}\right) .
$$

This is a stochastic process with statistics dependent on the arrival process, power allocation and channel distribution. We assume that under any power allocation, $P_{\text {sum }}(t) \rightarrow P_{\text {sum }}$ in distribution as $t \rightarrow \infty$, where $P_{\text {sum }}$ is a random variable with the steady-state distribution. We consider a stochastic constraint on the total power. Specifically, $\operatorname{Pr}\left(P_{\text {sum }}>\bar{P}\right) \leq q_{0}$, where $q_{0}>0$ is a small constant.

The resource allocation problem can be formally stated as

\section{Problem MAXU:}

$$
\begin{array}{ll}
\underset{P: \mathcal{H} \mapsto \mathbb{R}^{+}}{\operatorname{maximize}} & \lambda \mathbb{E}_{H}\{\tilde{U}[P(h)]\} \\
\text { subject to } & \operatorname{Pr}\left(P_{\text {sum }}>\bar{P}\right) \leq q_{0} .
\end{array}
$$

\section{Utility Based Power Allocation}

\section{A. Power Constraint Decomposition}

Let $\delta h$ be a small constant such that $h_{\max }-h_{\min }=K \delta h$, for some integer $K$. For $i=0, \ldots, K$, define $h_{i}=h_{m i n}+i \delta h$. For $i=0, \ldots, K-1$, let $N(i)$ be a random variable representing the number of active users in steady-state with channel gain in $\left[h_{i}, h_{i+1}\right)$. The steady-state total power, $P_{\text {sum }}$, can then be approximated as:

$$
P_{\text {sum }} \approx \sum_{i=0}^{K-1} P\left(h_{i}\right) N(i) \text {. }
$$

Since arrivals are Poisson with overall rate $\lambda$, then $N(i)$ is approximately the occupancy of an $M / G / \infty$ queue with arrival rate $\lambda f_{H}\left(h_{i}\right) \delta h$ and service time $T\left(h_{i}\right)$. Therefore, $N(i)$ is approximately Poisson distributed, and so $\mathbb{E}(N(i)) \approx$ $\operatorname{Var}(N(i)) \approx \lambda f_{H}\left(h_{i}\right) \delta h T\left(h_{i}\right)=\bar{N}\left(h_{i}\right) \delta h$, where $\bar{N}\left(h_{i}\right)=$ 
$\lambda f_{H}\left(h_{i}\right) T\left(h_{i}\right)$. Taking expected values, and letting $\delta h \rightarrow 0$, we have

$$
\mathbb{E}\left(P_{\text {sum }}\right)=\int P(h) \bar{N}(h) d h .
$$

Likewise, assuming $N(i), i=0, \ldots, K-1$ are independent, the variance of $P_{\text {sum }}$ is given by:

$$
\operatorname{Var}\left(P_{\text {sum }}\right)=\int P^{2}(h) \bar{N}(h) d h .
$$

For a large number of active users, we approximate $P_{\text {sum }}$ by a Gaussian random variable, yielding,

$$
\operatorname{Pr}\left(P_{\text {sum }}>\bar{P}\right)=Q\left(\frac{\bar{P}-\int P(h) \bar{N}(h) d h}{\sqrt{\int P^{2}(h) \bar{N}(h) d h}}\right) \leq q_{0},
$$

where $Q(x)=\int_{x}^{\infty} \frac{1}{\sqrt{2 \pi}} \exp \left(-\frac{t^{2}}{2}\right) d t$ is the complementary cumulative distribution function (c.d.f.) of the standard Gaussian random variable.

This can be simplified to

$$
\int P(h) \bar{N}(h) d h+k_{1} \sqrt{\int P^{2}(h) \bar{N}(h) d h} \leq \bar{P},
$$

where $k_{1}=Q^{-1}\left(q_{0}\right)$.

Since $\bar{N}(h)=\lambda f_{H}(h) T(h)$, we have:

$$
\int P(h) \bar{N}(h) d h=\lambda \mathbb{E}_{H}(E(h))
$$

and

$$
\int P^{2}(h) \bar{N}(h) d h=\lambda \mathbb{E}_{H}(P(h) E(h))
$$

where $E(h)=P(h) T(h)$ is the energy allocated to a user with channel gain $h$. An inactive user uses zero energy.

Substituting (7) and (8) into (6), constraint (3) can be approximated by:

$$
\lambda \mathbb{E}_{H}(E(h))+k_{1} \sqrt{\lambda \mathbb{E}_{H}(P(h) E(h))} \leq \bar{P}
$$

Finally, this can be further decomposed into three parts:

$$
\begin{cases}\lambda \mathbb{E}_{H}(E(h)) \leq \mathcal{E} & \text { average energy } \\ \mathbb{E}_{H}(P(h) E(h)) \leq G & \text { average power } \times \text { energy } \\ \mathcal{E}+k_{1} \sqrt{\lambda G} \leq \bar{P} & \text { tradeoff between } \mathcal{E} \text { and } G\end{cases}
$$

We will refer to Problem MAXU when (3) is replaced with (10) as Problem MAXUA. A solution to Problem MAXUA is provided next. We proceed in two steps. First, the utility maximizing power assignment is found subject to the first two constraints in (10) for given values of $\mathcal{E}$ and $G$. Next, the combination of $\mathcal{E}$ and $G$ that yields the highest utility rate is derived.

\section{B. Solution with Fixed $\mathcal{E}$ and $G$}

Given values of $\mathcal{E}$ and $G$, consider the following problem:

\section{Problem P1:}

$$
\begin{array}{cl}
\underset{P: \mathcal{H} \mapsto \mathbb{R}^{+}}{\operatorname{maximize}} & \lambda \mathbb{E}_{H}\{\tilde{U}[P(h)]\} \\
\text { subject to } & \lambda \mathbb{E}_{H}(E(h)) \leq \mathcal{E} \\
& \mathbb{E}_{H}(P(h) E(h)) \leq G .
\end{array}
$$

To gain insight into this problem, we first consider each of the constraints separately. First, we examine the problem with only the energy constraint, i.e.,

\section{Problem P2:}

$$
\begin{array}{ll}
\underset{P: \mathcal{H} \mapsto \mathbb{R}^{+}}{\operatorname{maximize}} & \lambda \mathbb{E}_{H}\{\tilde{U}[(P(h)]\} \\
\text { subject to } & \lambda \mathbb{E}_{H}(E(h)) \leq \mathcal{E} .
\end{array}
$$

From (4) and (7), $\mathbb{E}\left(P_{\text {sum }}\right)=\lambda \mathbb{E}_{H}(E(h))$, so that Problem P2 is equivalent to constraining the average sum power.

To continue, we assume that the transmission rate is proportional to the received power, i.e.,

$$
\mathcal{C}(h P(h))=k_{0} h P(h),
$$

where $k_{0}$ is a given constant. ${ }^{\dagger}$ It follows directly from (13) that the energy consumed by a user depends only on whether a user's transmission power is nonzero, and not on the specific power level, i.e.,

$$
E(h)= \begin{cases}P(h) T(h)=L / k_{0} h, & \text { for } P(h)>0 \\ 0, & \text { for } P(h)=0 .\end{cases}
$$

Since utility is strictly increasing in received power, it follows from (14) that the solution to Problem P2 is for each packet to be either denied transmission (blocked) or transmitted with infinite power. If no users are blocked and the energy constraint (11) is violated, then admission control is required to block some users. The choice of which users are blocked depends on whether $U(R)$ is bounded as $R \rightarrow \infty$. If $U(R)$ is bounded, the users requiring the highest energy should be blocked until (11) is satisfied. In this way the fewest users are blocked, and therefore (2) is maximized. If $U(R)$ is unbounded, the maximal utility rate is also unbounded, and which users are blocked is arbitrary as long as (11) is satisfied. In either case, $P_{\text {sum }}(t)=0$ with probability 1 and $P_{\text {sum }}(t)=\infty$ whenever a new request arrives. Of course, this is not realistic. This behavior is eliminated by adding constraint (12).

Next consider Problem P1 with only constraint (12):

Problem P3:

$$
\begin{array}{ll}
\underset{P: \mathcal{H} \mapsto \mathbb{R}^{+}}{\operatorname{maximize}} & \lambda \mathbb{E}_{H}\{\tilde{U}[P(h)]\} \\
\text { subject to } & \mathbb{E}_{H}(P(h) E(h)) \leq G .
\end{array}
$$

This is mathematically equivalent to the problem studied in [5]; as in [5], the solution can be attained via a pricing scheme.

Theorem 1: Consider the following pricing scheme: a channel dependent price per unit transmit power of the form $\alpha_{p}(h)=\alpha E(h)$ is announced; users respond by requesting power to maximize their surplus (utility minus cost), i.e.,

$$
P^{*}(h)=\arg \max _{P(h)}\{\tilde{U}[P(h)]-\alpha E(h) P(h)\} .
$$

If $\alpha$ is set such that (12) is satisfied with equality, this pricing scheme provides a power allocation that solves Problem P3.

This theorem can be easily proven using the Kuhn-Tucker optimality conditions. The set of active users and the assigned

\footnotetext{
$\dagger$ A linear relationship between rate and power is a reasonable approximation for many practical systems. For large enough rates, capacity considerations imply that this is optimistic.
} 
power levels are determined by $\alpha$, which can be interpreted as a fixed unit price on the product of power and energy. For each active user, the marginal utility with respect to power equals the price per unit power: $\frac{d \tilde{U}(P(h))}{d P(h)}=\alpha_{p}(h)$. Inactive users have lower marginal utility at zero than the price, i.e., $\left.\frac{d \tilde{U}(P(h))}{d P(h)}\right|_{P(h)=0}<\alpha_{p}(h)$. Since $\tilde{U}(P(h))$ is concave, $\frac{d \tilde{U}(P(h))}{d P(h)}$ is decreasing with $P(h)$. In other words, for inactive users, operating at any positive power gives a utility that is less than the cost (negative surplus). We call those inactive users intimidated due to a combination of high price and small initial slope of $\tilde{U}(P)$.

Assuming all users have the same $U(R)$ and that (13) holds, the set of users that are intimidated can be characterized as follows:

Theorem 2: There exists a threshold $h_{i}>0$ such that $P(h)>0$ if and only if $h>h_{i}$. The threshold $h_{i}$ satisfies:

$$
\left.\frac{d U(R)}{d R}\right|_{R=0}=\frac{\alpha_{p}\left(h_{i}\right)}{k_{0} h_{i}}
$$

The theorem follows easily from the fact that $\frac{d U(R)}{d R}=$ $\frac{d \tilde{U}(P)}{d P} \frac{d P}{d R}$ and that $\alpha_{p}(h)$ is decreasing in $h$. This theorem implies that a user with a low channel gain is penalized twice. First, this user requires more transmission power to achieve the same $S I N R$; second, the user is charged a higher unit price per power. Notice that as $G$ increases, $\alpha$ becomes smaller and $P(h)$ increases for all active users. This in turn increases the utility for each active user and hence results in a higher utility rate. Also notice that the constraint in Problem P3 does not depend on the traffic intensity $\lambda$, but only on the channel distribution, $f_{H}(h)$. It follows that changes in the arrival rate, for a fixed $f_{H}(h)$, will not effect the optimal price in Theorem 1 .

Now we return to Problem P1. The solution to this problem will be a combination of admission control, as in Problem $\mathrm{P} 2$, and the pricing approach from Theorem 1. The optimal combination of these approaches can be found in the following sequence:

1) Assume $P(h)>0$ for any $h$. Given $f_{H}(h)$ and (14), check the energy constraint (11). If it is violated, block users with channel gains $h \leq h_{e}$ where $h_{e}$ is selected such that constraint (11) is tight. Otherwise, admit all users. By convention, if (11) is satisfied with equality, we set $h_{e}=h_{\min }$. If (11) is loose, we set $h_{e}$ equal to any arbitrary value less than $h_{\text {min }}$.

2) Find $\alpha$ so that (12) is binding for the set of active users. The optimal power allocation is given by (15) for the active users. Blocked users are assigned zero power.

The reason users with the lowest channel gains are blocked is that with the same $U(R)$, these users always derive the lowest utility for any given $\alpha$. Therefore, there exists an energy induced cutoff threshold $h_{e}$ such that only users with $h \leq h_{e}$ are blocked via admission control. We note that at the optimum, (12) is always binding, whereas (11) may not be binding.

By formulating the problem with both constraints, the resource allocation is accomplished in two steps. The combination of the energy constraint and the arrival rate $\lambda$ may require admission control, i.e., some users may be blocked to satisfy the energy constraint. Among the remaining users, power levels are determined via pricing. Some users may also be intimidated depending on the price.

Admission control and intimidation are characterized by the channel gain thresholds, $h_{e}$ and $h_{i}$, respectively. We distinguish the following 3 cases.

C1: $h_{e} \geq h_{\min }$ and $h_{e} \geq h_{i}$. (Active users are determined by $h_{e}$.)

C2: $h_{\min }>h_{e}$ and $h_{\min } \geq h_{i}$. (All users are active.)

C3: $h_{i}>h_{e}$ and $h_{i}>h_{\min }$. (Active users are determined by $h_{i}$.)

\section{Optimal Admission Control/Pricing Trade-off}

Given $\mathcal{E}$ and $G$, we have shown that the optimal solution to Problem P1 consists of a combination of admission control and pricing. Returning to Problem MAXUA, notice that any pair of values $\mathcal{E}$ and $G$ that satisfy

$$
\mathcal{E}+k_{1} \sqrt{\lambda G} \leq \bar{P}
$$

results in a solution to Problem P1 that is also a feasible power allocation for Problem MAXUA. To solve Problem MAXUA, we want to find the combination that maximizes the utility rate. It is sufficient to consider values of $\mathcal{E}$ and $G$ such that (16) is tight. Since if $\mathcal{E}+k_{1} \sqrt{\lambda G}<\bar{P}$, we can always increase $G$ to the point where (16) is binding. A larger $G$ allows a lower $\alpha$, and therefore a higher utility rate.

Theorem 3: Consider P1 with constraints $(\mathcal{E}, G=$ $\left.\frac{1}{\lambda}\left(\frac{\bar{P}-\mathcal{E}}{k_{1}}\right)^{2}\right)$. As $\mathcal{E}$ increases from 0 to $\bar{P}$, the optimal solution transitions through the cases $\mathrm{C} 1, \mathrm{C} 2, \mathrm{C} 3$ in one of the following sequences: $\mathrm{C} 1 \rightarrow \mathrm{C} 2 \rightarrow \mathrm{C} 3$ or $\mathrm{C} 1 \rightarrow \mathrm{C} 3$.

Proof: Let A1 denote the set of values of $\mathcal{E}$ for which the optimal solution to $\mathrm{P} 1$ is in $\mathrm{C} 1$. Define A2 and A3 similarly. At $\mathcal{E}=0, h_{e}=h_{\max }$ and $h_{i}=0$; therefore $0 \in \mathrm{A} 1$. As $\mathcal{E}$ increases, $G$ decreases; this results in $h_{e}$ decreasing with $\mathcal{E}$ and $h_{i}$ increasing. This implies that if $\mathcal{E} \in \mathrm{A} 1$ then $\mathcal{E}^{\prime} \in \mathrm{A} 1$ for all $\mathcal{E}^{\prime} \leq \mathcal{E}$ and likewise, if $\mathcal{E} \in \mathrm{A} 3$ then $\mathcal{E}^{\prime} \in \mathrm{A} 3$ for all $\mathcal{E}^{\prime} \geq \mathcal{E}$. When $\mathcal{E}=\bar{P}, G=0$, in which case $h_{i}=\infty$, thus, $\bar{P} \in \mathrm{A} 3$. Therefore, the only possible sequences are $\mathrm{C} 1 \rightarrow \mathrm{C} 2 \rightarrow \mathrm{C} 3$ or $\mathrm{C} 1 \rightarrow \mathrm{C} 3$, which of these occurs depends on $h_{\min }$.

As noted previously, the constraint (12) is tight under an optimal power allocation. If the energy constraint (11) is loose, then $\mathcal{E}$ can be decreased, allowing for a larger $G$ without violating (16). This results in a higher utility rate. Therefore we have the following:

Theorem 4: The power allocation which solves Problem MAXUA satisfies both (11) and (12) with equality.

Corollary: The optimal $\mathcal{E}^{*} \in \mathrm{A} 1$, and $G^{*}=\frac{1}{\lambda}\left(\frac{\bar{P}-\mathcal{E}^{*}}{k_{1}}\right)^{2}$.

A1 is the only region where both constraints are tight. In A2 or A3, the energy constraint is always loose.

\section{Numerical Results}

In this section, we present numerical results to illustrate the ideas from the previous section. Throughout this section, users are assumed to have the same utility function:

$$
U(R)=1-e^{-R}
$$

and the channel distribution is assumed to be given by:

$$
f_{H}(h)=\frac{1}{4} h^{-\frac{5}{4}}, \text { for } h \in(1, \infty)
$$




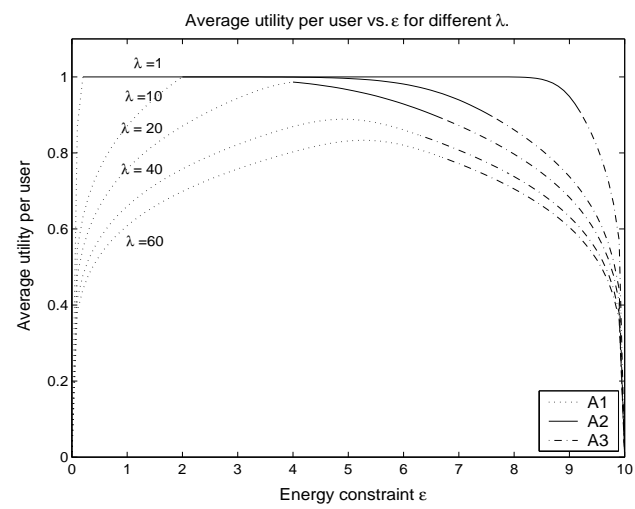

Fig. 2. Average utility per user vs. $\mathcal{E}$ for different arrival rates, $\lambda$.
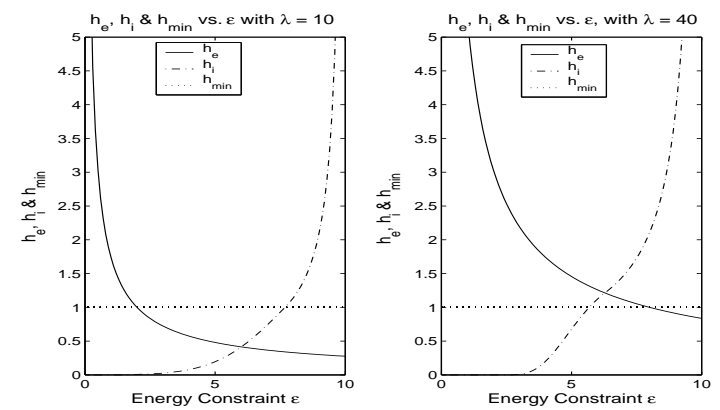

Fig. 3. $h_{e}, h_{i}$ vs. $\mathcal{E}$ for different $\lambda$ 's.

This is based on a distance-based attenuation formula $h(r)=$ $r^{-4}$ where users are distributed uniformly along the radius within a cell, i.e., $f_{r}(r)=1, r \in(0,1)$. We assume the file length is normalized so that $L=k_{0}$, which is the linear scaling factor between transmission rate and received power. In other words, one unit of received power results in one unit completion time.

Fig. 2 shows how the average utility per user varies with $\lambda$ and $\mathcal{E}$ (and therefore $G$ ) when $\bar{P}=10$ and $q_{0}=0.01$. The classification of the resulting allocation is indicated on the figure. Notice that the maximum point is always in A1 as predicted. Also observe that as $\mathcal{E}$ increases, the solution transitions from $\mathrm{C} 1 \rightarrow \mathrm{C} 2 \rightarrow \mathrm{C} 3$ when $\lambda$ is small $(\lambda=1,10,20)$. As $\lambda$ increases, the allocation transitions directly from $\mathrm{C} 1$ to $\mathrm{C} 3$ $(\lambda=40,60)$.

Fig. 3 shows how $h_{e}$ and $h_{i}$ vary with $\mathcal{E}$ under different arrival rates, $\lambda$. The minimum channel gain $h_{\text {min }}=1$ is also shown. For $h_{e}<h_{\min }$, we choose $h_{e}$ to satisfy $\lambda \int_{h_{e}}^{h_{\max }} \frac{1}{4} h^{-\frac{5}{4}} \frac{L}{k_{0} h} d h=\mathcal{E}$ so that the curve is extended continuously from where $h_{e} \geq h_{\text {min }}$. Observe that, as expected, $h_{e}$ decreases with $\mathcal{E}$ and $h_{i}$ increases. When $\lambda=10$, the system transitions from $\mathrm{C} 1$ to $\mathrm{C} 2$ when $h_{e}$ falls below $h_{m i n}$, and from C2 to C3 when $h_{i}$ increases above $h_{\min }$. When $\lambda=40$, the intersection point of $h_{e}$ and $h_{i}$ is larger than $h_{m i n}$; in this case the solution transitions directly from $\mathrm{C} 1$ to $\mathrm{C} 3$.

Fig. 4 shows the maximum average utility per user, $\mathbb{E}_{H}\{\tilde{U}[P(h)]\}$ versus the threshold $q_{0}$ for different arrival rates. As $\lambda$ increases, the average utility per user decreases; however, the overall utility rate $\lambda \mathbb{E}_{H}\{\tilde{U}[P(h)]\}$ increases. A smaller $q_{0}$,

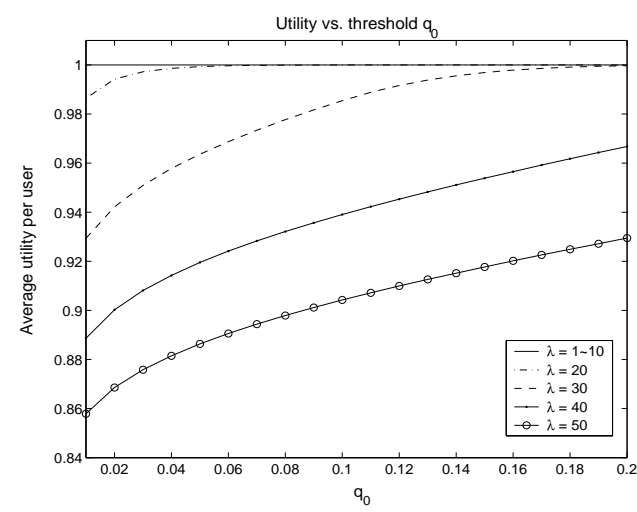

Fig. 4. Maximum $\mathbb{E}_{H}\{\tilde{U}[P(h)]\}$ vs. threshold $q_{0}$ for different $\lambda$ 's.

or equivalently a tighter power constraint, results in a lower utility per user. Notice the average utility per user is insensitive to $q_{0}$ when $\lambda$ is small $(<10)$. This is because the utility function we use $\left(U(R)=1-e^{-R}\right)$ is relatively flat (close to 1$)$ when $R$ becomes large. When $\lambda$ is small, the optimal power allocation lets users operate in the range of $R$ where the utility function is relatively flat.

\section{CONCLUSions}

We have studied forward link power allocation for stochastically varying traffic within a single cell. We use a stochastic total power constraint in order to allocate resources at a slow rate relative to the dynamics of the traffic. Specifically, power assignments depend only on the users' channel state, utility function and the long-range traffic statistics. We give an approximation for the power constraint that results in three tractable constraints, and show that a combination of admission control and pricing of power maximizes time-averaged utility rate. We categorize the tradeoff between admission control and pricing induced intimidation into three cases and show that the solution is always in case 1. Numerical results illustrate the tradeoff and show that for small $\lambda$, the derived utility rate is insensitive to the choice of $\mathcal{E}$ over a wide region.

\section{REFERENCES}

[1] N. Feng, N. Mandayam, and D. J. Goodman, 'Joint power and rate optimization for wireless data services based on utility functions", In Proc. CISS 1999, Johns Hopkins University, March 1999.

[2] S. Kalyanasundaram and M. Needham and R. Agrawal, 'Utility functionbased optimal resource allocation with a mixture of reallocation-tolerant and reallocation-intolerant users", In Proc. of SPIE, vol. 4531, Aug. 2001.

[3] S. Kunniyur and R. Srikant, 'End-to-End Congestion Control Schemes: Utility functions, Random Losses and Marks", In Proc. INFOCOM 2000, pp. 1323-1332, 2000.

[4] F. Kelly, "Charging and rate control for elastic traffic," European Trans. on Telecommunications, 8:33-37, 1997.

[5] P. Liu, M. Honig and S. Jordan, 'Forward-Link CDMA Resource Allocation Based on Pricing", Proceedings IEEE Wireless Communications and Networking Conference, 2000.

[6] J. Mo and J. Walrand, "Fair end-to-end window-based congestion control," In Proc. SPIE '98 International Symposium on Voice, Video and Data Communication, Boston, MA, Oct. 1998.

[7] S. Shenker, "Fundamental design issues for the future internet," IEEE Journal on Selected Areas in Communications, vol.13, pp. 1176-1188, 1995.

[8] L. Song and N. B. Mandayam, "Hierarchical SIR and rate control on the forward link for CDMA data users under delay and error constraints," IEEE Journal on Selected Areas in Communications, 19(10):1871-1882, October 2001. 